\title{
Miranda
}

Revue pluridisciplinaire du monde anglophone /

Multidisciplinary peer-reviewed journal on the English-

speaking world

$14 \mid 2017$

Early American Surrealisms, 1920-1940 / Parable Art

\section{Henri Durel, Francis Bacon et l'affirmation d'une science nouvelle en Angleterre}

\section{Claire Guéron}

\section{OpenEdition}

\section{Journals}

Édition électronique

URL : http://journals.openedition.org/miranda/9944

DOI : 10.4000/miranda.9944

ISSN : 2108-6559

Éditeur

Université Toulouse - Jean Jaurès

\section{Référence électronique}

Claire Guéron, « Henri Durel, Francis Bacon et l'affirmation d'une science nouvelle en Angleterre », Miranda [En ligne], 14 | 2017, mis en ligne le 03 avril 2017, consulté le 16 février 2021. URL : http:// journals.openedition.org/miranda/9944 ; DOI : https://doi.org/10.4000/miranda.9944

Ce document a été généré automatiquement le 16 février 2021.

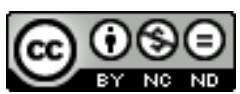

Miranda is licensed under a Creative Commons Attribution-NonCommercial-NoDerivatives 4.0 International License. 


\title{
Henri Durel, Francis Bacon et l'affirmation d'une science nouvelle en Angleterre
}

\author{
Claire Guéron
}

\section{RÉFÉRENCE}

Durel, Henri, Francis Bacon et l'affirmation d'une science nouvelle en Angleterre, Clermont-

Ferrand, Presses Universitaires Blaise Pascal, 2016, 267 pages, ISBN : 10 2-84516-722-9

1 Comme l'indique le terme d'«affirmation» contenu dans le titre, cet ouvrage s'intéresse à l'activité épistémologique plutôt qu'à l'activité scientifique de Francis Bacon. Avec cette distinction en tête, Henri Durel cherche dans ce traité à rendre compte d'un paradoxe et à dissiper un malentendu. Le paradoxe réside dans le fait que Francis Bacon, reconnu d'abord par la Royal Society puis par la communauté scientifique au sens large comme «le père de la science moderne", n'a jamais rien découvert, ni jamais rien inventé. Quant au malentendu, qui persiste en particulier en France à cause de l'influence de l'Encyclopédie des Lumières, c'est celui qui fait de Bacon le pourfendeur de l'obscurantisme religieux dans son élaboration d'une science moderne. Ces deux objectifs conduisent l'auteur à analyser l'oeuvre de Bacon comme un dialogue permanent avec les Écritures et avec la logique aristotélicienne. Tout au long de cette étude, Durel s'évertuera à démontrer que Bacon a posé les jalons d'une science nouvelle en dissociant savoir profane et savoir religieux, tout en affirmant vigoureusement la primauté de la connaissance révélée sur la connaissance issue de l'activité humaine. Cette rupture épistémologique lui permettra d'affirmer que le champ de la connaissance humaine est infini sans s'exposer à l'accusation d'impiété. Mais si cette approche ouvre la voie à l'envol des sciences expérimentales, Bacon, faute d'outils méthodologiques et d'instruments de mesure adéquats, n'a pas lui-même été 
capable de percer les secrets de la nature, c'est à dire de mettre à jour les lois physiques qui s'y manifestent.

Henri Durel consacre les trois premiers chapitres de l'ouvrage aux études suivies par Bacon à l'université de Cambridge, et aux conséquences de cet apprentissage sur sa pensée. A l'époque où les écrits d'Aristote dominent le cursus universitaire et même les écrits des théologiens, Bacon expose les limites de la dialectique aristotélicienne, mode de raisonnement replié sur lui-même, sans prise avec la nature. Il rejette de la même manière les Sommes de la théologie médiévale visant à faire la synthèse du christianisme et de la logique aristotélicienne pour aboutir à une science unique et englobante. Dans The Advancement of Learning (1605), Bacon pose le principe de base de la « dichotomie absolue entre la philosophie divine (...) et la théologie révélée »(p.55). $C^{\prime}$ est ce principe émancipateur qui permettra à la science nouvelle de se déployer.

Dans le quatrième chapitre, intitulé «Bacon promoteur de la botanique en Angleterre ", Durel nous montre un Bacon tournant le dos à l'abstraction aristotélicienne pour se confronter à l'expérience pratique du terrain. Les observations qu'il fait alors des techniques de greffe et de pollinisation artificielle l'amènent à constater la possibilité d'une symbiose entre technique et nature, et donc à rejeter l'opposition aristotélicienne entre le naturel et l'artificiel. Outre qu'il consacre la rupture entre science expérimentale et logique aristotélicienne, cet intérêt pour la botanique renforce chez Bacon l'idée d'une caution divine accordée à la curiosité humaine : «la posture du botaniste agenouillé devant le végétal [ne rappelle-t-elle pas] celle du chrétien devant le créateur »? (p.70).

4 C'est cette caution divine que Bacon va s'attacher à démontrer dans ses écrits, tout particulièrement dans The Advancement of Learning et sa version latine augmentée, De Dignitate et Augmentis Scientiarum (1623). Henri Durel consacre les chapitres 5 à 11 de son traité à la défense du savoir humain à laquelle se livre Bacon, Écritures à l'appui. L'auteur montre en effet que Bacon réussit le tour de force consistant à puiser dans la Bible, dont le livre de la Genèse semble pourtant établir clairement le caractère illicite de la curiosité humaine, les arguments de son plaidoyer en faveur de la recherche scientifique. En confrontant les différentes Bibles en circulation au début du dixseptième siècle, principalement la Vulgate, la Bible de Genève et la Bible des Évêques (pourtant hostile au savoir), et en retenant dans chacune d'elles les tournures qui abondent dans son sens, Bacon cherche à prouver que les Écritures n'interdisent en aucune façon, et même encouragent, la recherche de la connaissance. Tout en soulignant le caractère peu scientifique de la démarche consistant à ne retenir dans un échantillon que les résultats conformes à l'hypothèse de départ, Durel fait admirer l'exhaustivité et la minutie de ce travail philologique et herméneutique.

Les chapitres 12 à 14 reviennent sur l'idée de progrès. Durel montre que Bacon met la lacune au centre de son argumentation, le progrès étant avant tout conçu comme le fait de combler un vide. Ce progrès serait rendu possible, et même quasi-inévitable, par la structure de l'esprit humain, formaté par Dieu pour recevoir la connaissance du monde créé dont il est le miroir. A cet égard, Bacon anticipe la philosophie transcendantale de Kant, avec ses formes a priori de la sensibilité (perception). On retiendra de ces chapitres le formidable optimisme de Bacon pour qui la science permettra à l'homme de renouer avec la connaissance intuitive des choses que possédait Adam avant la chute. 
6 Le dernier chapitre, intitulé «Bacon n'est pas le fondateur de la science moderne en Grande-Bretagne : pourquoi? » revient sur les limites de l'approche baconienne, dont il a brièvement été question au début de l'ouvrage. Durel identifie deux obstacles principaux à l'évolution de la pensée baconienne vers une véritable méthode scientifique. En premier lieu, l'induction, processus permettant de remonter à la cause à partir de l'observation de ses effets, reste chez Bacon inféodée à la logique aristotélicienne, car elle est centrée sur la forme, c'est à dire les concepts, plutôt que sur la recherche des lois qui régissent les phénomènes naturels. Durel explique en partie cette faillite par un deuxième obstacle, à savoir la méconnaissance des mathématiques, que Bacon a très peu étudiées. Ainsi, Bacon, qui ignore tout du repère orthonormé et des équations, ne dispose pas des outils conceptuels qui lui permetttraient par exemple d'établir une corrélation entre la dilatation d'un corps et sa température.

7 Dans ce traité méthodique et fouillé, Henri Durel développe une argumentation étayée et originale, où l'accent est mis sur les sources bibliques du plaidoyer de Bacon en faveur des sciences profanes. L'explication détaillée que donne Durel de la méthode exégétique du philosophe, avec de nombreux tableaux à l'appui, peut surprendre par son côté systématique, qui rappelle la méthodologie des sciences dures, mais cette rigueur s'avère indispensable à la compréhension d'une démarche où l'essentiel est dans les détails. L'exemple du verset de la Genèse où il est question de l'inimitié entre Ève et le Serpent (Gn.3.15) est probant. Durel commence par citer un passage de The Advancement of Learning où Bacon résume (pour ensuite mieux les réfuter) les arguments des théologiens hostiles au savoir: «le savoir a en lui quelque chose du Serpent, et donc [...] là où il entre dans un homme, il le fait enfler; scientia inflat » (p. 112). Durel fait remarquer que cette formulation n'est pas conforme à la Vulgate, où il n'est jamais question de la morsure du serpent, mais seulement du "piège » qu'il tend aux enfants d'Ève. Ce décalage peut sembler anodin au lecteur, et ne pas justifier les douze pages de commentaire qui suivent, où l'auteur rapporte sa longue quête de la source de la citation (une note marginale dans la Bible de Genève). Mais on comprendra plus loin l'importance de ce passage : le "gonflement » associé à la morsure du serpent permet en effet à Bacon de passer de l'idée d'arrogance humaine à celle de plénitude ou de croissance, et ainsi de justifier le progrès.

8 Hernri Durel réussit donc son pari, en montrant que la foi chrétienne de Bacon sert de fondement à son engagement en faveur de la science profane, mais aussi que des lacunes en mathématiques empêchent le philosophe anglais d'être lui-même l'orfèvre de cette science. En lisant cet essai, le lecteur ne peut qu'apprécier l'effort de pédagogie déployé par l'auteur, qui, à l'aide de tableaux, de métaphores simples et de mises au point ponctuelles, parvient à rendre son exposé accessible, sans pour autant trahir la complexité de la pensée de Bacon. Si ce souci d'intelligibilité entraine quelques redites, celles-ci ont l'avantage de faire apparaître clairement les idées-forces du propos. Dans ce traité érudit et rigoureux, Henri Durel fait découvrir au lecteur un Bacon audacieux et novateur mais, malgré son dégoût pour les « idoles » de la pensée reçue, respectueux des textes sacrés. Si ces qualités peuvent paraître contradictoires à notre époque, toute la force de cet ouvrage réside dans l'habileté de l'auteur à démontrer la grande cohérence de la pensée de cette figure fondatrice de la science moderne. 
INDEX

Mots-clés : science, épistémologie, botanique, nature, mathématiques, religion, foi, Bible, théologie, progrès

Keywords : science, epistemology, botany, nature, mathematics, religion, faith, Bible, theology, progress

\section{AUTEURS}

\section{CLAIRE GUÉRON}

Maître de conférences

Université de Bourgogne Franche-Comté

claire.gueron@u-bourgogne.fr 